\title{
Nuevos Patrones de Reconocimiento Visual en el Ciclo Cardiaco
}

\author{
Estela Audelo ${ }^{1}$, Fernando Beristain ${ }^{2}$ y Carlos Vázquez ${ }^{1}$ \\ Universidad Nacional Autónoma de México, Facultad de Estudios Superiores Cuautitlán, \\ (1) Centro de Cómputo, (2) Departamento de Ingeniería y Tecnología, 57740 Cuautitlán Izcalli, \\ Edo. México-México (e-mail: \{audelovu, ferber, carlos\}@servidor.unam.mx)
}

\begin{abstract}
Resumen
El objetivo de este trabajo es aplicar el análisis no lineal en el estudio del ciclo cardiaco. Se analizaron 30 series de tiempo con diferentes tipos de ritmo cardiaco (10 de sujetos en ritmo sinusal normal, 10 de sujetos con fibrilación atrial y 10 de sujetos con arritmia supraventricular), con las herramientas de análisis computacional, de la teoría del caos y la dinámica no lineal, para conocer la complejidad del sistema y encontrar patrones de reconocimiento visual. Se encontró que el ciclo cardiaco es un sistema con un comportamiento dinámico mixto (periódico-caótico) y que las representaciones en el espacio-fase (atractores), obtenidas a partir de la relación entre la señal original $(\mathrm{x}(\mathrm{t}))$ y la primera derivada, pueden ser utilizadas como patrones de reconocimiento visual en la identificación de los tres ritmos cardiacos estudiados.
\end{abstract}

Palabras clave: ciclo cardiaco, patrones de reconocimiento visual, teoría del caos, dinámica no lineal, atractor

\section{New Patterns of Visual Recognize in the Cardiac Cycle}

\section{Abstract}

Time series (30) with different heart rate were analyzed (10 of subjects in normal sinus rhythm, 10 of subjects with atrial fibrillation and 10 of subjects with supraventricular arrhythmia), with analysis computational of chaos and nonlinear dynamics theory in order to know the complexity of system and find visual recognize patterns. It was found that cardiac cycle is a mixed dynamic behavior system (periodic-chaotic) and the representations (attractors) in space phase obtained from the relation between the original signal $(x(t))$ and first derived, can be used like visual recognize patterns in the identification of the three studied heart rhythms.

Keywords: cardiac cycle, visual recognize patterns, chaos theory, nonlinear dynamics, attractor 


\section{INTRODUCCIÓN}

El ciclo cardiaco como sistema dinámico se ha venido estudiando, desde hace aproximadamente tres décadas, con las herramientas de la teoría del caos (Savi, 2005; Wessel et al., 2007). En el Instituto Tecnológico de Massachussets el físico Richard J. Cohen y sus colegas diseñaron una simulación por computadora de los ritmos cardiacos y descubrieron que la duplicación de periodos es un indicio de la proximidad de un ataque cardiaco (Briggs y Peat, 1991); en Harvard el profesor Ary Goldberger y colaboradores han realizado estudios del intervalo del ritmo cardiaco (RR) y la variabilidad de la frecuencia cardiaca (VFC) utilizando las herramientas de la teoría del caos, comparando sujetos saludables con sujetos enfermos (Pincus y Goldberger, 1994); detectando apnea (Mietus et al., 2000); contrastando dos técnicas de meditación (Peng et al., 1999). Los resultados de los estudios realizados por el equipo del profesor Goldberger (2002a, 2002b), han demostrado que una alta variabilidad (caos) se presenta en el corazón cuando es aún joven y saludable y a medida que éste va envejeciendo o está próximo a alguna arritmia esta variabilidad tiende, a un comportamiento periódico.

En un estudio sobre el efecto de la postura corporal en la variabilidad del ritmo cardiaco de sujetos sanos, de diferente edad, Vuksanovic y Gal (2005), encontraron que solo la postura tiene un efecto significativo en las propiedades no lineales. Llabrés et al. (2005), estudiaron la dinámica del electrocardiograma (ECG) de sujetos con y sin miedo a volar, descubriendo que las señales del ECG de todos los sujetos presentan propiedades no lineales. Al-Fahoum y Qasaimeh (2006), desarrollaron un algoritmo para clasificar arritmias del ciclo cardiaco, basándose en la distribución del atractor en el espacio-fase reconstruido. Srinivasan et al. (2003), propusieron una técnica para clasificar arritmias cardiacas, basada en el análisis del espacio-fase. Kikuchi et al. (2006), encontraron diferencias entre los atractores del ritmo cardiaco de fetos sanos y fetos con crecimiento limitado.

La teoría del caos no hace énfasis en el desorden del sistema, sino precisamente en el orden que exhibe; establece que los sistemas dinámicos no lineales y complejos son inherentemente impredecibles pero al mismo tiempo garantiza que, a menudo, la mejor manera de definir el comportamiento de estos sistemas, es a través de las representaciones gráficas en el espacio-fase.

El objetivo del presente trabajo es aplicar el análisis no lineal en el estudio del ciclo cardiaco para demostrar que la representación de los atractores en el espacio-fase puede ser un buen auxiliar en la detección de los cambios de ritmo cardiaco.

\section{METODOLOGÍA}

Se evaluaron 10 series de tiempo de sujetos en ritmo sinusal normal, 10 de sujetos con fibrilación atrial y 10 de sujetos con arritmia supraventricular; las series de tiempo fueron obtenidas de las bases de datos de la página PhysioNet del Centro Nacional de Salud (Goldberger et al., 2000).

Se eligió para este estudio la señal de la derivación II (por ser la más próxima a la dirección de la despolarización ventricular y como los ventrículos poseen la mayor masa muscular proporcionan la mayor corriente y por lo tanto el voltaje más elevado) transformando los archivos al formato de datos necesario para el análisis; de esta manera se obtuvieron 10 series de tiempo de sujetos en ritmo sinusal normal con 7,680 datos cada una, 10 series de tiempo de sujetos con fibrilación atrial con 15,000 datos cada una y 10 series de tiempo de sujetos con arritmia supraventricular con 7,680 datos cada una. Las 30 series de tiempo fueron analizadas con los programas Chaos Data Analyzer ${ }^{\circledR}$ (Sprott, 1998) y Visual Recurrence Analysis ${ }^{\circledR}$ (Kononov, 2006) con el fin de caracterizar el comportamiento de los registros cardiacos.

Se examinó la representación en el espacio-fase de la relación entre la señal original y la primera derivada, localizando el núcleo del atractor y midiendo el ángulo de inclinación, el cual se obtiene trazando las tangentes a cada una de las orbitas que convergen al núcleo, después se traza la bisectriz del ángulo generado por las tangentes y se mide el ángulo de la línea base hasta la bisectriz. 


\section{RESULTADOS Y DISCUSIÓN}

Se analizaron estadísticamente los registros obtenidos, determinando que no existen diferencias significativas entre los tres tipos de sujetos; concluyendo que, estos resultados no aportan información que permita diferenciar los comportamientos del ritmo cardiaco (tabla 1). A través del análisis del espectro de frecuencia se estableció que todas las series de tiempo presentan un comportamiento predominantemente periódico; el análisis del histograma muestra que las series de tiempo estudiadas no presentan comportamiento estocástico y se determinó que las gráficas de distribución de probabilidad no son de utilidad para distinguir entre los diferentes tipos de sujetos.

Tabla 1: Valores promedio de los índices estadísticos

\begin{tabular}{|l|c|c|c|c|c|c|c|}
\hline \multicolumn{1}{|c|}{ Registro } & Mínimo & Máximo & Mediana & Varianza & $\begin{array}{c}\text { Desviación } \\
\text { estándar }\end{array}$ & $\begin{array}{c}\text { Recurrencia } \\
\%\end{array}$ & $\begin{array}{c}\text { Determinismo } \\
\%\end{array}$ \\
\hline $\begin{array}{l}\text { Ritmo sinusal } \\
\text { normal }\end{array}$ & -0.807 & 1.809 & -0.041 & 0.090 & 0.279 & 64.06 & 84.64 \\
\hline $\begin{array}{l}\text { Fibrilación } \\
\text { Auricular }\end{array}$ & -2.280 & 2.910 & 0.054 & 0.256 & 0.506 & 76.48 & 93.57 \\
\hline $\begin{array}{l}\text { Fibrilación } \\
\text { Supraventricular }\end{array}$ & -2.322 & 1.411 & -0.125 & 0.208 & 0.407 & 65.35 & 85.94 \\
\hline
\end{tabular}

Los gráficos de recurrencia visual (Kononov, 2006) de los tres tipos de sujetos, muestran pequeñas diferencias en estructura pero no son lo suficientemente notables como para poder ser utilizados en la práctica clínica como patrones de reconocimiento visual.

Por otro lado, al reconstruir el sistema, en el espacio-fase, a partir de graficar la relación entre la señal original y la misma señal original con un retardo $n$ establecido $(n=1,2$ y 4$)$, se encontró que la representación del atractor cambia en función del retardo tal como se muestra en la Fig. 1. Debido a estos cambios, esta representación no tiene un uso práctico como herramienta auxiliar en el estudio del ECG.
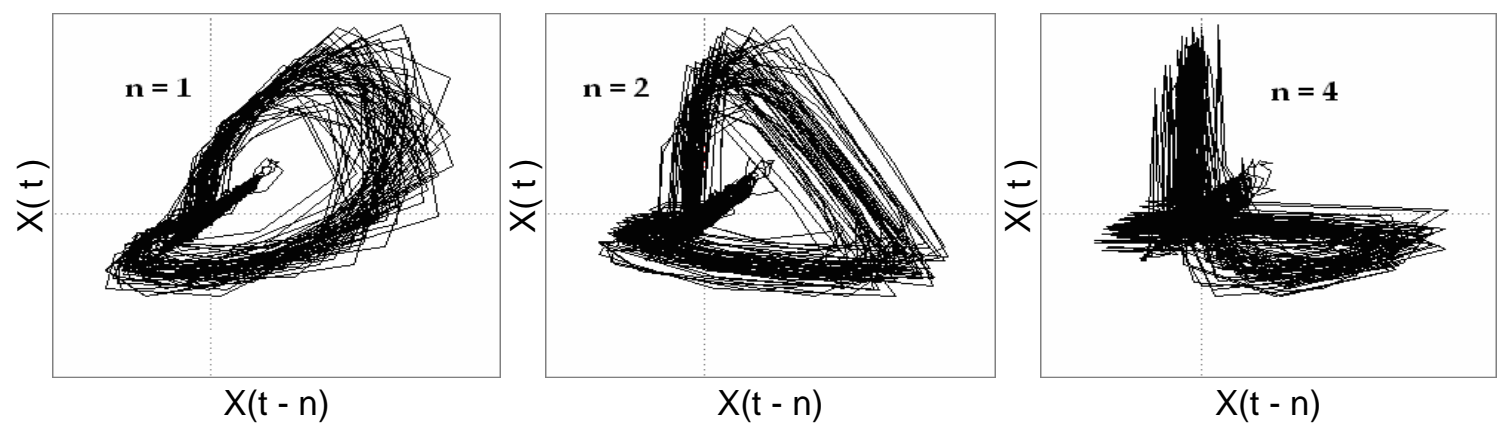

Fig. 1: Representación del atractor al cambiar el retardo para un mismo registro

Sin embargo, la representación en el espacio-fase de la relación entre la señal original y la primera derivada, muestra que el atractor obtenido solo se ve afectado por el comportamiento dinámico del ritmo cardiaco; de acuerdo a esto, al analizar el gráfico se observó que existe una asociación significativa entre el ritmo cardiaco y el atractor extraño que representa las ondas $P, Q R S, y$ del ECG el cual presenta un comportamiento cuasi-periódico, aparentando ciclos límite sobrepuestos con una zona densa a la que se denominó núcleo del atractor, en la Fig. 2 se identifica al núcleo con la letra A.

Asimismo, de acuerdo a la forma de los atractores de los registros de sujetos en ritmo sinusal normal y registros de sujetos con fibrilación atrial se identificaron tres casos:

1. El núcleo, al formarse, se enlaza con el cuerpo del atractor formando una curva en forma de "V" (Fig. 3 y 4 ).

2. El núcleo al formarse enmascara la "V" (Fig. 5 y 6).

3. El núcleo está separado de la "V", esta forma sólo se da en los registros de sujetos con fibrilación atrial (Fig. 7). 


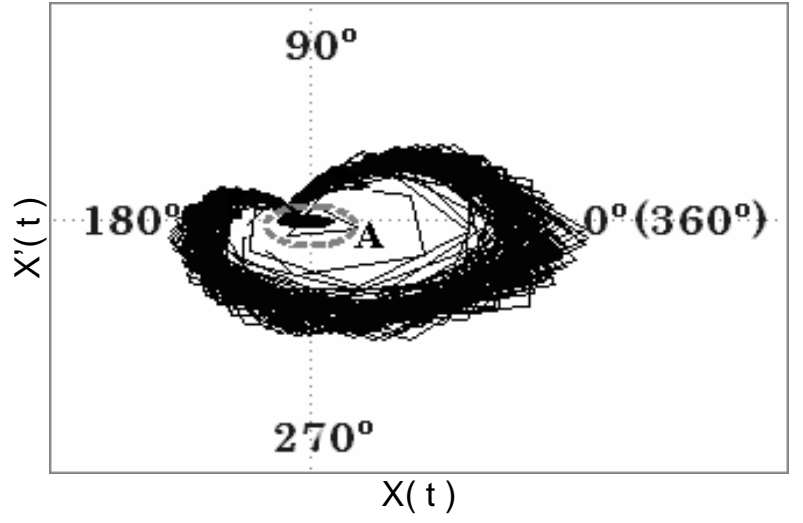

Fig. 2: Núcleo del atractor

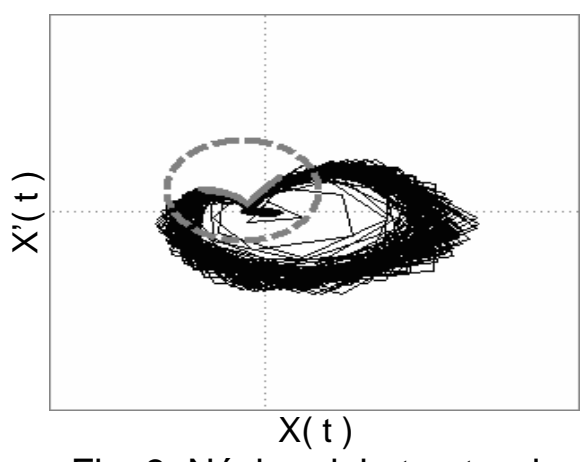

Fig. 3: Núcleo del atractor de sujeto en ritmo sinusal normal

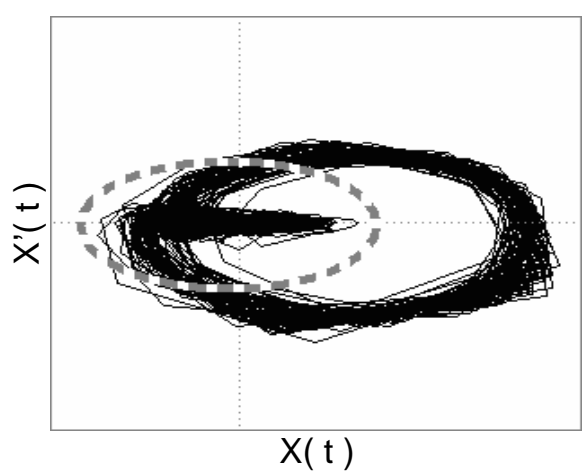

Fig. 5: Núcleo del atractor de sujeto en ritmo sinusal normal

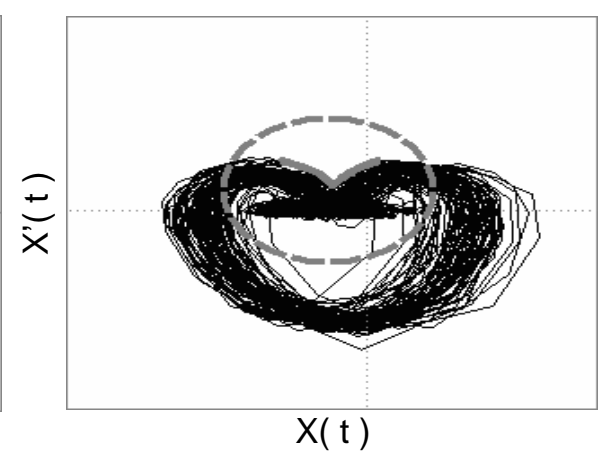

Fig. 4: Núcleo del atractor de sujeto con fibrilación atrial

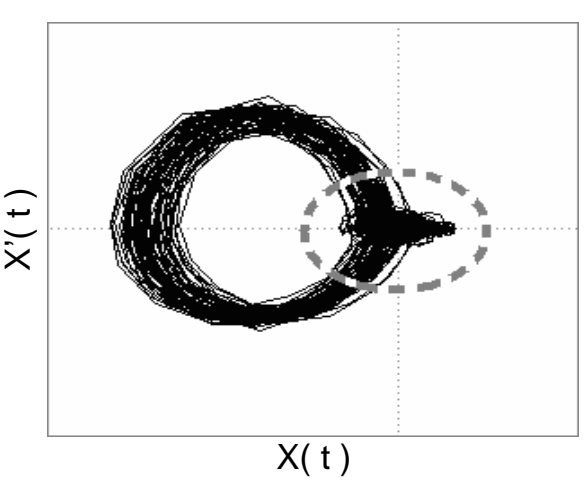

Fig. 6: Núcleo del atractor de sujeto con fibrilación atrial

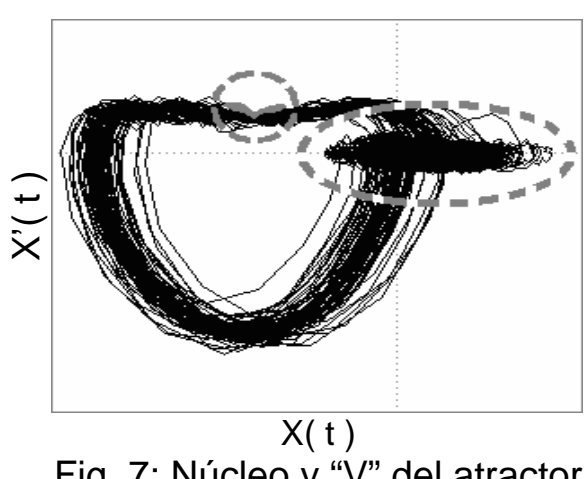

Fig. 7: Núcleo y "V" del atractor de sujeto con fibrilación atrial

De acuerdo al análisis de los tres casos arriba mencionados, se observa que el núcleo se encuentra en el origen de coordenadas y que su posición forma un ángulo de inclinación que permite diferenciar a los sujetos en ritmo sinusal normal de los sujetos con fibrilación atrial ; dicho ángulo (Fig. 8) se mide trazando las tangentes a cada una de las curvas que forman la "V", después se traza la 
bisectriz del ángulo $\theta$, generado por las tangentes (tg) y, por último, se mide el ángulo $\phi$ de la línea base (LB) hasta la bisectriz, definiendo de esta forma, el ángulo de inclinación del núcleo; esta metodología fue aplicada para analizar los casos 1 y 3. Cuando no es posible identificar la "V" como en el caso 2, se toma directamente como parámetro de identificación, la posición en la que se encuentra el núcleo con respecto a la línea base.

En el caso 3 la identificación se puede establecer de acuerdo a la medición del ángulo de inclinación o en función de la posición del núcleo.

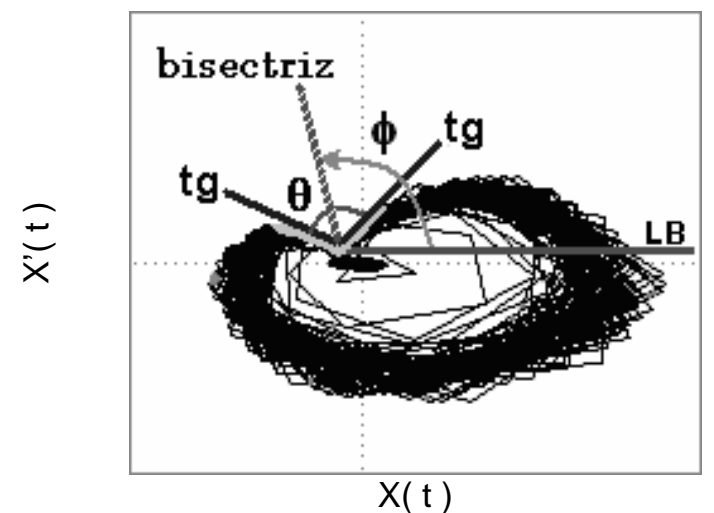

Fig. 8: Forma de medir el ángulo de inclinación del núcleo

De acuerdo a los resultados obtenidos de la medición, se encontró que los registros de sujetos en ritmo sinusal normal, presentan un ángulo de inclinación del núcleo entre $105^{\circ}$ y $180^{\circ}$; mientras que, los sujetos con fibrilación atrial muestran un ángulo de inclinación entre $0^{\circ}$ y $90^{\circ}$.

El núcleo de los atractores de las series de sujetos con arritmia supraventricular, es más complejo por lo que es difícil medir el ángulo, razón por la cual no se utilizó para identificar este tipo de registros; para la identificación del comportamiento del ritmo cardiaco de estos sujetos se utilizó la forma del atractor, ya que, contrario a lo que se establece en la literatura, se encontró por este método que sí tienen estructura; la forma con líneas más largas y rectas dando la apariencia de que el atractor fue recortado, es distintiva de estos registros (Fig. 9).
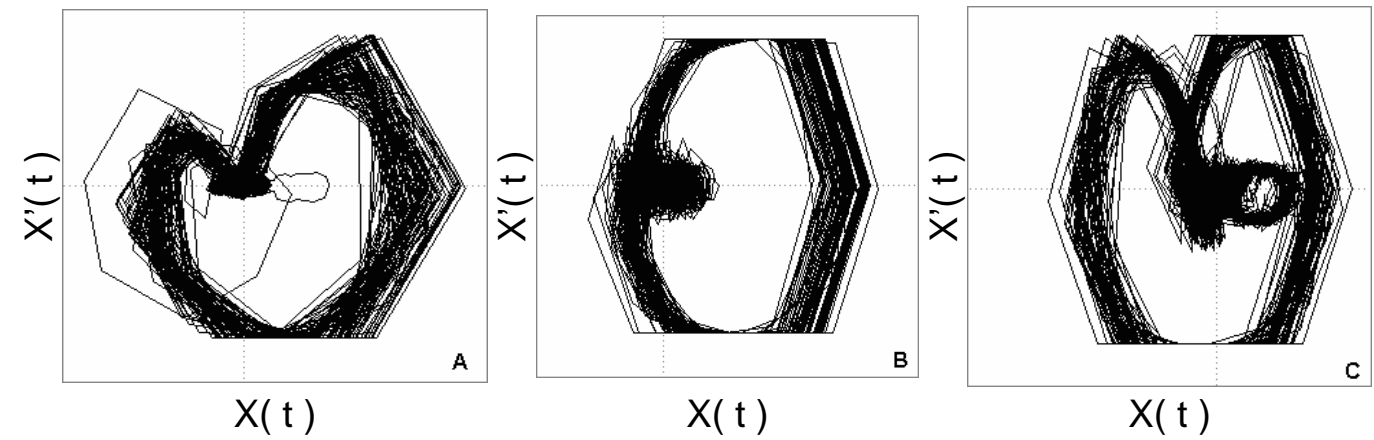

Fig. 9: Atractores de tres sujetos con arritmia supraventricular

\section{CONCLUSIONES}

En el presente trabajo se comprobó, a lo largo de los diferentes análisis realizados, que el ciclo cardiaco es un sistema con un alto grado de periodicidad; que al reconstruir el sistema de origen al graficar x(t) vs. $x^{\prime}(\mathrm{t})$, se obtuvieron atractores extraños cuasi-periódicos con apariencia de ciclos límite sobrepuestos, mostrando una región densa a la que se denominó núcleo del atractor, éste presenta diferencias en el ángulo de inclinación, lo cual permitió contrastar entre registros de sujetos en ritmo sinusal normal y registros de sujetos con fibrilación atrial.

En los registros de sujetos con arritmia supraventricular el ángulo de inclinación del núcleo no permitió una identificación convincente, en cambio, la forma de sus atractores con líneas más largas y rectas, dando la apariencia de un atractor recortado, es distintivo de estos registros. 


\section{REFERENCIAS}

Al-Fahoum, A.S. y A.M. Qasaimeh; ECG Arrhythmia Classification Using Simple Reconstructed Phase Space Approach, Computers in Cardiology, 33, 757-760 (2006).

Briggs, J. y D. Peat; Espejo y Reflejo: del Caos al Orden, $1^{\text {ra }}$ ed., Consejo Nacional de Ciencia y Tecnología, México (1991).

Goldberger, A.L. y otros 5 autores; Fractal Dynamics in Physiology: Alterations with Disease an Aging, Proceedings of the National Academy of Sciences USA, 99, 2466-2472 (2002a).

Goldberger, A.L., C.K. Peng y L.A. Lipsitz; What is Physiologic Complexity and How does it Change with Againg and Disease? Neurobiology of Aging ISSN 0197-4580, 23, 23-26 (2002b).

Goldberger, A.L. y otros 9 autores; New Research Resource for Complex Physiologic Signals (en línea), 20 abril de 2005, http://www.physionet.org/physiobank/, American Heart Association, Cambridge, MA. USA (2000).

Kikuchi, A. y otros 6 autores; Nonlinear Analyses of Heart Rate Variability in Normal and Growthrestricted Fetuses, Early Human Development ELSEVIER, 82, 217-226 (2006).

Kononov, E.; Visual Recurrence Analysis, v4.9, software de computadora en disco (April 2006 Released).

Llabrés, J. y otros cuatro autores; ¿Caos en el Electrocardiograma de Estudiantes con Miedo a Volar? Un Análisis de No Linealidad, International Journal of Clinical and Health Psychology ISSN 1697-2600, 5(2), 273-284 (2005).

Mietus, J., C.K. Peng, P.C. Ivanov y A.L. Goldberger; Detection of Obstructive Sleep Apnea from Cardiac Interbeat Interval Time Series, Computer in Cardiology, 27, 753-756 (2000).

Peng, C.K. y otros 6 autores; Exaggerated Heart Rate Oscillations During Two Meditation Techniques, International Journal Cardiology, 70(2), 101-107 (1999).

Pincus, S.M. y A.L. Goldberger; Physiological Time Series Analysis: What does Regularity Quantify. American Journal of Physiology, 266(4), H1643-H1656 (1994).

Savi, M.A.; Chaos and Order in Biomedical Rhythms, J. Braz. Soc. Mech. Sci. \& Eng. (en línea). 27(2), 157-169 (2005). [citado 2007-06-14], Disponible en:<http://www.scielo.br/scielo.php? script= sci_arttext\&pid=S1678-58782005000200008 \&lng=en\&nrm=iso>. ISSN1678-5878.

Sprott, J.C.; Chaos Data Analyzer, v2.1, software de computadora en disco, University of Wisconsin (1998)

Srinivasan, N., M.T. Wong y S.M. Krishnan; A New Phase Space Analysis Algorithm for Cardiac Arrhythmia Detection, Proceedings of the $25^{\text {th }}$ Annual International Conference of the Institute of Electrical and Electronics Engineers, Engineering in Medicine and Biology Society, 82-85 (2003).

Vuksanovic, V. y V. Gal; Nonlinear and Chaos Characteristics of Heart Period Time Series: Healthy Aging and Postural Change, Autonomic Neuroscience: Basic and Clinical, 121, 94-100 (2005).

Wessel N., J. Kurths, W. Dito y R. Bauernschmitt; Introduction: Cardiovascular Physics, Chaos, 17, 015101 (2007). 